\title{
A Survey on Multi-Scale Medical images Fusion Techniques: Brain Diseases
}

\author{
Ahmed Sabeeh Yousif, Zaid Bin Omar, Usman Ullah Sheikh \\ School of Electrical Engineering, University Technology Malaysia \\ 81300, Johor Bahru, Malaysia \\ syahmed@graduate.utm.my
}

\begin{abstract}
Brain diseases such as degenerative (alzheimer's disease), neoplastic disease (brain tumor like sarcoma, glioma) are considered an interesting topic areas in the medical image fusion diagnosis. Pixel-level image fusion techniques are designed to combine multiple/multi-scale input images into a fused image, which is expected to be more informative for human or machine perception as compared to any of the input images. Since they are difficult to be summarized ; survey paper are characterized by (1) medical image definition, brain diseases challenges, analysis a various techniques for multi-scale image fusion with its own modalities, fusion rule, fusion strategy and dis-advantage ,Whilst used a database of medical images for medical Harvard School (brain diseases) which contains various groups of co-registered multi-modal images including MRI/CT, MRI/PET and PET/SPECT and MRI (T1/T2) images.
\end{abstract}

Keywords: Image fusion, Brain Diseases Challenges, Multi-scale medical images fusion methods.

\section{Introduction}

Over the past several decades' diseases have fallen before the scythe of human intelligence in the form of biomedical advances. Medical images used in the application of brain disease diagnosing and treatment since last two decades. Due to a more diseases inside whole brain are related to human lives like (stroke "brain attacks), so the researchers put all efforts to access to better and high accurate diagnosis by apply the computer vision and image processing as one of technology solution today for explore precisely abnormal part in human brain by employed the phenomena of fusion process for multiple sensors with different modalities of medical imaging [1].

Data fusion is the combination of different types of data to obtain more information from the merged data instead of considering each dataset separately [2]. It can be used to produce new raw data or more informative new data based on the original data. Once the new data are generated, it is often expected to be more supportive in decision-making process than using the original datasets. The data form of image fusion is the image containing brightness, color, informative, edge, structure of organs visibility and other scenery features, which can be given in the form of a picture or a series of images.

Multi-model medical image fusion is merging of multiple image/sensors or multiple imaging modalities. The main target of the medical image fusion is to improve imaging quality with preserving the specific

DOI: $10.14738 /$ jbemi.71.7415

Publication Date: $29^{\text {th }}$ February 2020

URL: http://dx.doi.org/10.14738/jbemi. 71.7415 
Ahmed Sabeeh Yousif, Zaid Bin Omar, Usman Ullah Sheikh; Title: A Survey on Multi-Scale Medical images Fusion Techniques: Brain Diseases. Journal of Biomedical Engineering and Medical Imaging, Volume 7, No 1, February (2020), pp 18-38

features and minimize randomness and redundancy for maximize the clinical applicability of images for diagnosis and assessment of medical issues. Today, medical image fusion was consider a main solution to overcome medical issues reflected through images of human body, organs, and cells since it contains a various range teqinques medical images fusion and information fusion .Medical images modalities mainly concerned on Ultrasound Guided Imaging (USG), Computed Tomography (CT), and Magnetic Resonance Imaging (MRI) along with functional MRI (fMRI), Positron Emission Tomography (PET), and Single-Photon Emission Computed Tomography (SPECT) [3-12] as also illustrated in figure.1. These sensors support us complementary information about patient's pathology, anatomy, and physiology and specially for brain disease, For example, CT is widely used for tumor and anatomical detection, whereas information about soft tissues is obtained by MRI. Similarly, other medical imaging techniques like fMRI (functional magnetic resonance imaging), PET (positron emission tomography), SPECT (single positron emission computed tomography) provide functional and metabolic information. Further, T1-MRI image provides details about anatomical structure of tissues, whereas T2-MRI image gives information about normal and abnormal tissues [13].

\section{Related work}

There have been several studies touched medical image fusion issues from different perspectives. Some of them are mentioned. Pradeep K. and M. Hossain entitled by "multimodal fusion for multimedia analysis: a survey" in 2010 [14]. It provides a survey study for some topics that discuss the pros of multimodal fusion and the big issues that may appear with five main aspects: level of fusion, how, when ,what to fuse, methods of multimodal fusion for all rule based schemes, classification schemes, and the estimation schemes. S.L. Jany Shabu and C. Jayakumar also wrote paper on "Survey of Image Fusion Techniques for Brain Tumor Detectio" in 2013 [15], and they went through medical exiting medical images fusion that used Genetic algorithm to detect the brain tumor by extracting feature such as extraction of color, texture and shape features. Alex James and Belur also presented a review study entitled by "medical image fusion: a survey of the state of the art" in 2014 [16]. This study involved imaging modalities, fusion algorithms and also the human body parts interested (organs) that used for medical image fusion. These topics are consolidated with a large number of analogous studies in similar subjects and they conclude that medical image fusion is moving towards to be adapted by the clinical application and treatment verification in the coming next years. Furthermore in 2014 [17], a survey study for image fusion methods that applied for medical field is presented by K.P.Indira and R.Rani Hemamalini to explained methods use in fusion process such as curvelet transform, wavelet transform, contourlet transform, stationary wavelet transform, and framelet transform. Multiple researches have discussed the subject of medical image fusion in different perspectives [18,19]. Wu D., Yang A., Zhu L., Zhang C. offered in 2014 also[20] "Survey of Multi-Sensor Image Fusion" and they focused on image fusion algorithm at all levels of fusion, and then makes the summary and comparison of these algorithms. Fatma El-Zahraa ElGamal and Mohammed Elmogy is offered in 2015 [21] a review study of "current trends in medical image registration and fusion". They focused on image fusion steps, registration steps, registration challenges, fusion process are introduced to square up to the further studies that ameliorate medical image registration. Swathi.P.S, Sheethal.M.S and Vince Paul wrote on "Survey on Multimodal Medical Image Fusion Techniques" in (2016) [22], This paper has centered on the many image fusion techniques on the wavelet generation methods with different fusion rule based on it and also NSCT, Contourlet Transform with its contributed on the effectives on the performance of transform based vision fusion methods 
outcome due to artifacts color, resolution. Authors also gave deep look on the existing methods in term of merit and demerits. Bhavana. V and Krishnappa. H.K wrote a survey paper" A Survey on Multi Modality Medical Image Fusion" in (2016) [23], they explained with details the modalities that had been used in different techniques for family of wavelet methods with fusing various modalities (MRI, CT, PET, SPECT) of brain images in medical field into a distinct image with more detailed anatomical information and high spectral information is highly desired in clinical diagnosis. Detailed survey concluded that all these techniques mentioned above have either a serious cons of color drawbacks, visual clarity or less informative in the gray matter area (GM) of the high-activity region of the fused image. Heba M. Elhoseny, El-Sayed Mahmoud El-Rabaie, Osama S. Farag Allah, Fathi E. Abd El-Samie wrote paper on " Medical Image Fusion: A Review Present Solutions and Future Directions" in (2017) [24], they had been covered the following: basic overview of the image fusion methods, applications, merits, and demerits of fusion procedures, A short explanation of radiological imaging modalities, usage, applications, and observed pros and cons of each modality is presented to provide a comprehensive view of medical imaging modalities. Also introduced some novel trends in the medical fusion filed. Bikash Meher, Sanjay Agrawal, Rutuparna Panda , Ajith Abraham offered survey paper" A survey on region based image fusion methods " in (2018) [25],they focused on state-of-the-art survey methods for region detection with concluded that ICA perform better outcome fused image in term of region detect, compression and evaluation with various metrics for different application was reviewed like medical image with brain dataset, standard images datasets.

\section{Contributions and organization of this article}

Despite existing reviews having summarized varieties of medical fusion methods, none of them focused on the fusion techniques for brain diseases data such as (Alzheimer's disease (AD), Parkinson's Disease (PD), brain attacks (stroke), Scroma), which brain currently consider the main challenge in the field of medical images diagnosing that related for the human lives. This survey provides a structured and extensive distinctive of medical images fusion based mutli-scale methods for retrieval more informative in the application brain diseases with narrow down the survey by focused on standard datasets available in the Harvard university medical center, USA with study compression between various techniques on different diseases with specific modalities (MRI,CT,PET,SPECT) [26], various modalities used in fusion process as follow: (CT/MRI,MRI/PET,MRI/SPECT, MRI (T1/T2). The reason for adapted Harvard datasets for this survey:

(1) All images were co-registered accurately with different modalities (CT, MRI, PET, and SPECT) that used by researchers in the field of enhanced brain images fusion processing in the clinical /treatment analysis and evaluation performance perfectly.

(2) It can show clearly the various types of diseases with different patients 'scenarios, for example ((Alzheimer's disease (AD) and brain attacks (stroke),) with highlight the specific abnormal part within brain like abnormal soft/hard tissues and abnormal cells.

In Section 4, we will provide a view of the limitation of brain diseases correlated with fusion process. Section 5, presents the application domain (brain diseases definition) with focus on Alzheimer's disease as widely used as database for fusion research development. Section 6, draw general view to readers by defines medical image fusion with mathematically expression. Section 7 , highlighted the categories of fusion level. Section. 8; elaborated multi-level medical images methods with explained the most metrics used in the evolution performance for multi-scale methods in the literatures. 
Ahmed Sabeeh Yousif, Zaid Bin Omar, Usman Ullah Sheikh; Title: A Survey on Multi-Scale Medical images Fusion Techniques: Brain Diseases. Journal of Biomedical Engineering and Medical Imaging, Volume 7, No 1, February (2020), pp 18-38

\begin{tabular}{|c|c|c|c|}
\hline Combination & Modality 1 & Modality 2 & Fused Image \\
\hline MRI-PET & & \\
\hline MRI-SPECT & & & \\
\hline MRI-CT & & \\
\hline
\end{tabular}

Fig.1. Examples of multi-modal medical image fusion for brain diseases (The fusion of MRI-CT, MRI-PET and MRI-SPECT images.) [9].

\section{Brain Diseases Challenges}

Every disease in the human brain has specific characteristics, tissues and generally artifacts like (noise, less resolution, low contrast, and redundant information) will damage totally the precise position of abnormal cells for various modalities that doctor/radiology looking for detect and accurate information about diagnose [27].

Radiologists in particular are required to read more and more cases with more and more images per case [28- 35]. Shortages in radiologists especially for example specialists in rural and medically underserved areas compound the problem. Physicians are working longer hours than ever before, and concerns have been raised regarding fatigue and whether it adversely affects diagnostic accuracy.

There are broad range of methods of medical image fusion were designed to address the medical images challenges imitated from captured images of the human body parts, organs, and tissues and cells. There are huge applications of the image fusion techniques in the historical analysis and medical diagnostics, multimodal image fusion is another method used for medical imaging applications. By multi modal means images with different modalities: CT and MRI scan [30,31], visible, or ultraviolet, etc. The prime goal of the multimodal fusion is to decrease the amount of data for emphasizing on the band specific information. As with time more medical image data is acquired and resulted many mystery to doctors and investigators [32], as how to merge huge amount data and abstract higher quality information for users, how to get rid of data redundancy, etc. The fusion technique, with a data fusion to organizes, connects, and combines multiple source and multi temporal data, gives the powerful tool for these data processing problems

A complete robust and accurate image fusion scheme for application of brain diseases usually includes the following components corresponding to major steps of brain characterization process performed by a radiologist:

(1) To quantify of lesions, diseases and/or regions of interest for the brain.

(2) Lesions, diseases and/or region of interest characterization including specific feature extraction and decision making on the degree of malignancy and further course of action.

(3) High accurate outcome brain medical images in term of (free noise images, visual quality of organs and tissues) are radiologist target. 
Moreover, medical imaging modalities incompetents for some of application in medical diagnosis and in totally will effect for clinical analysis for patient. The issues with these alternative imaging modalities are as follows: (1physicains/radiologist: exposing the patient's body to the radiations that are harmful to the patient's health. (2: images manipulation: like artifacts (noise, blur, less contrast).

This enforces to explore new fusion imaging technologies for combining information from multiple imaging modalities. The latter seems to be more meaningful because of lower cost and shorter time, compared with the former. The multi-modal medical image fusion traditionally centers on various categories: MRI-CT, MRI-PET and MRI-SPECT images fusion, as shown in figure 1.

In general ,brain diseases diagnose/treat considered hot topic today due to relevant with human live and body healthy and also it is main sensitive organ as it can control whole body, so the researcher put high effort in the literature review of computer vision and medical image processing like ( analysis, fusion, segmentation, classification, enhancements).in the fusion process, some of them focus on how to maximize the single image fused informative details [33,34], the other start looking for suppress the artifacts like noise[35,36,37] ,blur [38, 39,40]. The rest emphases on the how to preserved edge ,boundary, smoothly, sharpness $[41,42,43,44]$.from my point , brain disease diagnosing/treatment is critical work in the computer vision and cannot bear any Proportion of error in diagnosing diseases and that consider as lack and vary of fused medical image requirements in one time like (high visually, free noise, more informative, high contrast, edge preserved, accurate anatomy of tissues) in the previous work of medical image fusion algorithms for the application of brain diseases diagnosing and treatments[40-44] . In summary, the treatment of the diagnostic image data is performed by a physicist, who analyzes and aggregates them according to his knowledge. The aim is to provide a better medical decision, to propose a prognosis, or to assist physicians in a surgical intervention in the case of brain studies.

\section{Application domain(Brain)}

The Brain Research through Advancing Innovative Neuro technologies (BRAIN) is aimed at revolutionizing our understanding of the human brain. By accelerating the development and application of innovative technologies, researchers will be able to produce a revolutionary new dynamic picture of the brain that, for the first time, shows how individual cells and complex neural circuits interact in both time and space. Brain is one of the important organs that have more details like soft and hard tissues with small size that shown in imaging scan and therefore the researcher try to reduce the artifact for that images to read images with high visual quality due to brain consider main human organ body and effect on patient 's health. The imaging studies reveal several important pieces of information about the brain which are otherwise not visible to human sensory mechanisms. The most commonly used image modalities to study the brain include CT [45], MRI [46, 47], PET [48, 49], SPECT [50, 51].For example, Alzheimer's disease was founded 100 years ago. But during the past 30 years, only researches have been developing in its risk factors, symptoms, causes, and treatments. Nowadays throughout the world, more than 35 million people have been affected by Alzheimer's disease with its various stages [52-57].

\section{Medical fusion methods definition}

Image fusion in medical field may be considered as combining pertinent information from a series of images into one single informative and complete image than any input sensor images. More precisely, fusion is the integration of information from a set of registered images without the introduction of distortion $[58,59]$. 
Ahmed Sabeeh Yousif, Zaid Bin Omar, Usman Ullah Sheikh; Title: A Survey on Multi-Scale Medical images Fusion Techniques: Brain Diseases. Journal of Biomedical Engineering and Medical Imaging, Volume 7, No 1, February (2020), pp 18-38

It is observed from the state of arts that image fusion approaches are divided into two types, spatial based and transform based. In spatial based methods, the pixels of the images to be fused are merged in two types also, a linear or non-linear manner, and mathematically express as following:

$$
I_{f}=\emptyset\left(I_{1} I_{2}, \ldots \ldots, I_{M}\right)=\alpha_{1} I_{1}+\cdots, \alpha_{M} I_{M} \ldots \ldots \ldots \ldots
$$

Where $I_{1}, I_{2} \ldots, I_{M}$ is the inputs image sensor, $\varnothing$ denotes the fusion rule, $\alpha$ is a constant such that:

$$
\sum_{n=1}^{M} \alpha_{n}=1 \ldots \ldots \ldots \ldots
$$

In the other side, transform domain with complex algorithms observed from the literature with steps of convert the inputs images from different sensor to space domain such as Curvelet domain, wavelets domain or pyramids with aid of fusion rule for adding the high and low frequency and finally apply inverse transform to achieve reconstruction of original image. The outcome/fused image explained mathematically as follow:

$$
\left.I_{f}=M^{-1}\left(\varnothing\left(M\left(I_{1}\right), M\left(I_{2}\right), \ldots \ldots, M_{N}\right)\right)\right) \ldots \ldots
$$

Where $M$ is the forward transformation operator, $M^{-1}$ is the inverse transformation operator.

In terms of multi-modal medical image fusion, scheme for image decomposition and reconstruction closely relates to the quality extracted from the images. Characteristic of approaches in this frame aims at decomposing the original image into a sequence of images and then reconstructing the decomposition images into a single image. As mentioned in the previous works, five various key methodologies introduced: (1) color space [60,61], (2) pyramid [62,63] , (3) wavelet [64-73] with shown as multi-scale image decomposition and reconstruction, (4) sparse representation [74-78] and (5) salient feature [7981].

Table 1: Comparison of the five schemes of image decomposition and reconstruction.

\begin{tabular}{|c|c|c|c|c|c|}
\hline Scheme & $\begin{array}{c}\text { Salient } \\
\text { feature }\end{array}$ & $\begin{array}{c}\text { Sparse } \\
\text { representation }\end{array}$ & Color space & pyramid & Wavelet \\
\hline $\begin{array}{c}\text { Frequency } \\
\text { domain }\end{array}$ & no & yes & no & yes & yes \\
\hline Spatial domain & yes & yes & yes & no & no \\
\hline Multi-scale & yes & no & no & yes & yes \\
\hline $\begin{array}{c}\text { Scale } \\
\text { invariance }\end{array}$ & yes & no & no & no & yes \\
\hline Dictionary & no & yes & no & no & no \\
\hline Directive & no & no & no & no & yes \\
\hline
\end{tabular}

From Table 1, the comparison of algorithms for image decomposition and reconstruction with the indexes of spatial domain, frequency domain, multi-scale, scale invariance, dictionary and directive filter. Because the images existed in pseudo-color, color space fusion algorithm is used for multi-modal medical image fusion. Don't like other methods, color space algorithms are employed to process entire/inputs images in spatial domain. Then multi-scale decomposition (MSD) is employed to extract and merge salient features of medical images at different scales [82]. Algorithms based on multi-scale decomposition are wavelet, pyramid and salient feature. The cons of wavelet methods are scale invariance and directive filter. Add to, salient feature algorithms are employed according to the multi-scale decomposition tool in spatial 
domain. In addition, the sparse representation methods, inspired from the compressed sensing algorithms, construct a dictionary of input images.

\section{Categories of Fusion Levels}

Image Fusion has become a common term used within medical diagnostics and treatment. whenever multiple images of a patient are available, these are combined to get fused image. This fused image contains additional information than the combined individual images which helps in better diagnostics and treatment to the patient of various brain diseases affected[84,85,86].

There are three types of fusion strategies, low/pixel level, medium/features level, top/decision level. Pixel-level image fusion combines registered source images into a single fused image pixel by pixel and it works either on the raw pixels obtained from the imaging sensors or on the corresponding multiresolution transform coefficient [87]., An example of Pixel level would be the pixel averaging algorithm, where the mean values of the pixel-by-pixel is taken by inputs image as the fused image. Pixel-level image fusion, as mentioned above, is widely used in medical imaging of brain diseases [66], and computer vision $[88,83]$ due to the benefits of containing the original measured quantities easy implementation, and computationally efficiency[89]. Based on the adopted transform strategy, the existing image fusion methods can be categorized into four major families: 1) the multi-scale decomposition based methods such as pyramid[4], wavelet [90-92], complex wavelet [93], curvelet [94, 95]; 2) the sparse representation such as orthogonal matching pursuit[96, 97], group SR [98], gradient constrained SR [99], simultaneous OMP (SOMP) [100], joint sparsity model [101-103], SR with over-complete dictionary and structural subdictionary [104-106], spectral and spatial details dictionary [107];3) the methods which perform the fusion directly to the image pixels or in other transform domains such as the principal component [108]space or the intensity-hue-saturation color space[109-111]. 4) The methods combining multi-scale decomposition such as transform-SR [112], IHS- wavelet [113], sparse representation, principal component analysis, and other transforms. In addition to the signal transform scheme, the other key factor affecting fusion results is the fusion strategy. The fusion strategy is the process that determines the formation of the fused image from the coefficients or pixels of the source images.

Whilst Fusion at medium/feature level [114] means extract the desire feature from image, it first requires extraction of features from the source images (through e.g. segmentation); fusion then takes place based on features that match some selection criteria. Features are specific structures in the image such as points, edges or objects. The extraction of features involves detecting the edges of objects present in the images. After extracting the features fusion process combines all features of the source images. The fusion process is performed on the reduced selected features instead of entire image. The selected features are expected to contain the relevant information from the input image. This Feature-Level fusion is very useful when performing analysis on complex data. Wavelet Methods in Feature-Level Image Fusion [115] has proposed a region based approach for image fusion using Dual-Tree Complex Wavelet Transform (DT-CWT). The entropy of regions computed using the DT-CWT detail coefficients is used as the activity measure and regions are merged by selecting respective regions with maximum entropy. The Independent Component Analysis (ICA) bases are very efficient tools, which can replace common transform employed in image fusion, such as the Dual-Tree complex Wavelet Transform and can outperform wavelet based methods [116] As ICA has more degrees of freedom than DWT and DWFT, it depicts the image features more accurately. Different feature level ICA based fusion are available in literature[117]. Susmitha Vekkot, and Pancham Shukla [118] proposed a hybrid architecture for Wavelet based Image Fusion. The proposed 
Ahmed Sabeeh Yousif, Zaid Bin Omar, Usman Ullah Sheikh; Title: A Survey on Multi-Scale Medical images Fusion Techniques: Brain Diseases. Journal of Biomedical Engineering and Medical Imaging, Volume 7, No 1, February (2020), pp 18-38

hybrid architecture is the combination of advantages of pixel and region based fusion in a single image which can help the development of sophisticated algorithms enhancing the edges and structural details.

Finally, the Decision-Level of fusion is highest level/abstraction fusion with combining information from multiple systems to give fused information (graph description). [119,120.]. Decision level fusion in the brain diseases aims; to increase the diagnostic decision accuracy from the image beyond the levels derived by individual detection techniques. Each technique is employed to obtain a decision of detecting abnonnalities of an endoscopic: image by using associated features [121 ]. The decision fusion approach includes three steps: fusion, evaluation and learning. Fusion step is to combine sub-decisions derived independently from corresponding techniques to a final diagnostic decision. The supervised evaluation comes from the expert physician experience. In the learning step, the outcomes of the new case are fed back as learning data to the fusion step to recalculate the weight.

\section{Multi-scale decomposition based methods}

Multi-scale transform is a tool that has been shown to be very useful for image fusion and other image processing applications [122, 145]. In previous research, commonly researchers used multi-scale decomposition methods for image fusion in the low/pixel level due to easily implementation with verified high performance and accuracy diagnosing of different types of diseases compare to feature level or decision level algorithms. Visual quality ,suppress noise ,ignore blur ,contrast, edge preserve, still and became the interest topic and racing for the future trend for development medical image fusion algorithms. Pixel based multi-scale fusion methods are commonly used because of their simplicity for enhancing the image qualities. But there performance is not consistent with different kind of medical imaging environments. Therefore, it is required to improve the efficiency of these fusion methods.

Table .2; explained and analysis in details the various methods for multi-scale medical fusion with Medical imaging modalities and diseases, fusion rule, fusion strategy and disadvantages (cons) of algorithms were elaborated in details. Moreover, the experimental results are implemented on the image database from the Whole Brain Web Site of the Harvard Medical School which contains four groups of co-registered multi-modal images including MRI-CT, MRI-PET and PET-SPECT,MRI(T1-T2) images. The testing images have been used in many related paper [123, 124,125]. The platform is MATLAB R201xx.Moreover, the dataset mentioned above can implemented on various cases for patients for example; A 73 year old woman was brought to neurological evaluation imaging by MRI,PET by her brother because of a 3 year history of memory impairment. She had become lost on several occasions and had orienting himself in unfamiliar circumstances. This woman effected by the disease namely Alzheimer [134]. MRI,PET showed a globally widened hermispheric, which is more prominent in parietal lobes. regional cerebral metabolism is markedly abnormal. The objective performances of different methods are evaluated with various image fusion assessment metrics. The first simplest full-reference metrics based signal distortion defined with complex mathematical definition theory is involved with entropy (EN), difference of entropy (DEN), overall cross entropy (OCE), standard deviation (STD), sharpness (SP), RMSE, peak signal to noise ratio (PSNR)[126]. The second class [127] based on HVS is to measure the information of salient feature transferred from the input images to the fused image, such as SSIM, the phase congruency based index

$\left(Q_{G}\right)$, the gradient based index $\left(Q_{A B / F}\right)$, etc. It is used to compute the error between test and original images with less informative in salient information of gradient, contrast and edge between different image components. Another type of objective image quality based salient features, it tell you how well 
the salient features are transferred from the input images to the fused image, The metric $Q_{C F}[128]$ that embraces both contrast enhancement and image fusion to measure the performance of multi-modal medical image fusion algorithms. Natural image quality evaluator (NIQE) [129] is used as the no-reference objective quality assessment tool in medical image fusion and considered as blind image quality analysis [130]. Moreover, some other metrics based on signal distortion are inspired from the information theory, such as PSNR is widely used for objective quality, EN is indicates the amount of information in the fused image, DEN gave the difference of entropy between the input images and the fused image, OCE is reflects the entropy of two input images and fused image [131], visual information fidelity (VIF) denotes relationship between image information and visual quality during the distortion process [132], $\mathrm{MI}$ is focused on estimating the amount of information transferred from the input images into the fused image [133] and SF employed for measure the overall clarity level of the fused images [132]. However, the proposed method in this paper has some limitations in term of Blur and noise introduced due fusion steps, High computational time of perform the multi-level like NSCT, information of region interest limited with color information, blurry of informative edges, etc..) .

Finally, every methods presented in table.2 has own characterizes that depend directly to the type of decomposition methods, fusion rule ,fusion strategy to access to the target that researcher looking for, for example ; Garuav [134]proposed NSCT domain algorithm for enhance the details of the fused image and can improve the visual effect with less information distortion that the rest in previous work but still fall down in the noise issue. Jiao Du , Local[139] proposed Laplacian Filtering Domain(LLF) with limitations as: (1) LLF does not run as fast as other multi-scale tools. . (2) The proposed image fusion rule introduced less color information.

Table.2: Various techniques for multi-scale medical image fusion in the brain diseases application .

\begin{tabular}{|c|c|c|c|c|c|}
\hline No. & Authors \&Methods & $\begin{array}{l}\text { Modality\& } \\
\text { diseases }\end{array}$ & Fusion Rule & Fusion Strategy & Cons \\
\hline 1. & $\begin{array}{l}\text { Garuav, NSCT Domain } \\
\text {,2013[134] }\end{array}$ & $\begin{array}{l}\text { MRI/PET,M- } \\
\text { RI/SPECT. } \\
\text { Diseases: } \\
\text { Alzheimer } \\
\text { diseases,suba-cute } \\
\text { stroke,recurre-nt } \\
\text { tumor. }\end{array}$ & $\begin{array}{l}\text { Phase congruency- } \\
\text { cy and directive } \\
\text { contrast }\end{array}$ & $\begin{array}{l}\text { Low frequency fused by } \\
\text { phase congruency and } \\
\text { high frequency will fused } \\
\text { by directive contrast }\end{array}$ & $\begin{array}{l}\text { Noise introduced due } \\
\text { fusion steps also the } \\
\text { devices generated images. }\end{array}$ \\
\hline 2. & $\begin{array}{l}\text { Guocheng ,NSCT } \\
\text { Domain, } 2015 \\
\text { [135], }\end{array}$ & $\begin{array}{l}\text { MRI/PET,MR- } \\
\text { I/SPECT. } \\
\text { Diseases: } \\
\text { Alzheimer diseases. }\end{array}$ & $\begin{array}{l}\text { Standard deviation, } \\
\text { Shannon entropy } \\
\text { and weight maps. }\end{array}$ & $\begin{array}{l}\text { Low frequency } \\
\text { coefficients: standard } \\
\text { deviation and Shannon } \\
\text { entropy for super press } \\
\text { the contrast issues. } \\
\text { High frequency: weight } \\
\text { maps which are } \\
\text { determined by the } \\
\text { saliency maps }\end{array}$ & $\begin{array}{l}\text { High computational time } \\
\text { of perform the multi-level } \\
\text { decomposition .also less } \\
\text { color distortion } \\
\text { introduced. }\end{array}$ \\
\hline 3. & $\begin{array}{l}\text { JiaoDu, Laplacian } \\
\text { pyramid ,2016[136] }\end{array}$ & $\begin{array}{l}\text { MRI/CT,MRI/PET,M } \\
\text {-RI/SPECT. } \\
\text { Diseases: } \\
\text { Alzheimer }\end{array}$ & $\begin{array}{l}\text { Weighted } \\
\text { computing }\end{array}$ & $\begin{array}{l}\text { Weighted computing in } \\
\text { each scale with the aim } \\
\text { to enhance the outline } \\
\text { and colure contrast }\end{array}$ & $\begin{array}{l}\text { More blurry with MRI/CT in } \\
\text { the outcome fused images, } \\
\text { and high noisy introduced } \\
\text { with MRI/PET fused image. } \\
\text { Also more time cost for } \\
\text { overall running method. }\end{array}$ \\
\hline
\end{tabular}


Ahmed Sabeeh Yousif, Zaid Bin Omar, Usman Ullah Sheikh; Title: A Survey on Multi-Scale Medical images Fusion Techniques: Brain Diseases. Journal of Biomedical Engineering and Medical Imaging, Volume 7, No 1, February (2020), pp 18-38

\begin{tabular}{|c|c|c|c|c|c|}
\hline & & $\begin{array}{l}\text { diseases,suba-cute } \\
\text { stroke. }\end{array}$ & & & \\
\hline 4. & $\begin{array}{l}\text { Zhiqin, dictionary } \\
\text { learning approach, } 2016 \\
{[137]}\end{array}$ & $\begin{array}{l}\text { MRA/MRI- } \\
\text { T1,MRI/PET } \\
\text { Diseases: general }\end{array}$ & $\begin{array}{l}\text { Summation of } \\
\text { coefficient's } \\
\text { patches. }\end{array}$ & $\begin{array}{l}\text { Informative patch } \\
\text { obtained by patched } \\
\text { sampling scheme with } \\
\text { applied the summation } \\
\text { for patches clustering for } \\
\text { assign its to K-SVD } \\
\text { classifier. }\end{array}$ & $\begin{array}{l}\text { High computational time } \\
\text { with subjective analysis: } \\
\text { blurry of informative } \\
\text { edges. }\end{array}$ \\
\hline 5. & $\begin{array}{l}\text { Cheng-I chen, IHS and } \\
\text { Log -Gabor } \\
\text { transform,2017 [138] }\end{array}$ & $\begin{array}{l}\text { MRI/PET. } \\
\text { Diseases: } \\
\text { Alzheimer diseases. }\end{array}$ & $\begin{array}{l}\text { Maximum } \\
\text { selection (MS). }\end{array}$ & $\begin{array}{l}\text { Suitable decomposition } \\
\text { scale for MRI and } \\
\text { intensity component for } \\
\text { PET was perform, then } \\
\text { MS apply for the high } \\
\text { frequency sub-band and } \\
\text { two-stage fusion rule } \\
\text { based on weighted- } \\
\text { averaging scheme and } \\
\text { visibility measure for the } \\
\text { low-frequency sub-band } \\
\text { are employed llastly, } \\
\text { reverse log-Gabor } \\
\text { wavelet transform to the } \\
\text { fused high- and low- } \\
\text { frequency sub-bands. }\end{array}$ & $\begin{array}{l}\text { The outcome images are } \\
\text { more effective with noisy } \\
\text { (salt and paper )with less } \\
\text { subjective quality (more } \\
\text { blurry) }\end{array}$ \\
\hline 6. & $\begin{array}{l}\text { Jiao Du, Local Laplacian } \\
\text { Filtering } \\
\text { Domain(LLF),2017[139]. }\end{array}$ & $\begin{array}{l}\text { MRI/PET,MR- } \\
\text { I/SPECT. } \\
\text { Diseases: sub - } \\
\text { acute stroke. }\end{array}$ & $\begin{array}{l}\text { (LEM) local energy } \\
\text { maximum for } \\
\text { approximates } \\
\text { images, and } \\
\text { information of } \\
\text { interest (IOI) for } \\
\text { residual images. }\end{array}$ & $\begin{array}{l}\text { Fused } \\
\text { image }=\sum_{i=1}^{L-1} R_{F}^{i}+G_{F}^{L} \text {. } \\
\text { The fusion of the } \\
\text { approximate images and } \\
\text { the residual images } \\
\text { according to inverse LLF. }\end{array}$ & $\begin{array}{l}\text { 1-Less speed run than } \\
\text { other mutli-scal methods. } \\
\text { 2-information of region } \\
\text { interest limited with color } \\
\text { information. }\end{array}$ \\
\hline 7. & $\begin{array}{l}\text { Ebenezer, 2018[140], } \\
\text { wavelet based } \\
\text { homographic filter. }\end{array}$ & $\begin{array}{l}\text { MRI/PET,MRI/SPEC } \\
\text { T,MRI/CT,MRI(T1- } \\
\text { T2). } \\
\text { Diseases: } \\
\text { Alzheimer's } \\
\text { diseases and brain } \\
\text { tumor }\end{array}$ & $\begin{array}{l}\text { By direct add fused } \\
\text { adder } 1,2 \text { using } \\
\text { pixel based } \\
\text { averaging rule. }\end{array}$ & $\begin{array}{l}\text { The approximation and } \\
\text { details coefficients of } \\
\text { modality1 and modality } 2 \\
\text { are given to } \\
\text { adder1,adder } \\
\text { respectively ,later the } \\
\text { grey wolf optimization } \\
\text { will enhance the fusion } \\
\text { process. }\end{array}$ & $\begin{array}{l}\text { Focusing on the resolution } \\
\text { of fused image with discard } \\
\text { the transferred noise } \\
\text { through the process of } \\
\text { fusion and also edge } \\
\text { preserved. It has limited } \\
\text { when using in the } \\
\text { survalaince application due } \\
\text { to various luminance and } \\
\text { reflectance parameters. }\end{array}$ \\
\hline 8. & $\begin{array}{l}\text { Raja Lingam B,PCNN } \\
\text {,2018[141] }\end{array}$ & $\begin{array}{l}\text { MRI/PET,MR- } \\
\text { I/SPECT,MRI/CT. } \\
\text { Diseases: sub } \\
\text { acute stroke }\end{array}$ & Neural network. & $\begin{array}{l}\text { Perform segmentation } \\
\text { operation on the pre- } \\
\text { processed multimodality } \\
\text { medical image with the } \\
\text { PCNN. }\end{array}$ & $\begin{array}{l}\text { Less visual quality (PSNR) } \\
\text { compared with other } \\
\text { methods that mentioned in } \\
\text { the paper. }\end{array}$ \\
\hline
\end{tabular}




\begin{tabular}{|c|c|c|c|c|c|}
\hline 9. & $\begin{array}{l}\text { Jiao Du, parallel saliency } \\
\text { features ,2018[142] }\end{array}$ & $\begin{array}{l}\text { MRI-CBV/SPECT- } \\
\text { TC, MRI-T1/PET- } \\
\text { FDG.. } \\
\text { Diseases: } \\
\text { Alzheimer's } \\
\text { diseases }\end{array}$ & $\begin{array}{l}\text { 1- Activity level } \\
\text { measurement. } \\
\text { 2- Coefficient } \\
\text { grouping. } \\
\text { 3- Coefficient } \\
\text { combination. }\end{array}$ & $\begin{array}{l}\text { Multi-scale image } \\
\text { decomposition BY } \\
\text { averaging filter, then } \\
\text { fuse smooth layers and } \\
\text { detail layers using inter- } \\
\text { scale dependencies, } \\
\text { finally, calculate the } \\
\text { fused image using intra- } \\
\text { scale dependencies. }\end{array}$ & $\begin{array}{l}\text { Saliency features are only } \\
\text { used to measure the } \\
\text { activity levels of MRI, PET, } \\
\text { and SPECT images. } \\
\text { 2- Less visibility tissues in } \\
\text { term of contrast and color } \\
\text { resolution. }\end{array}$ \\
\hline 10. & 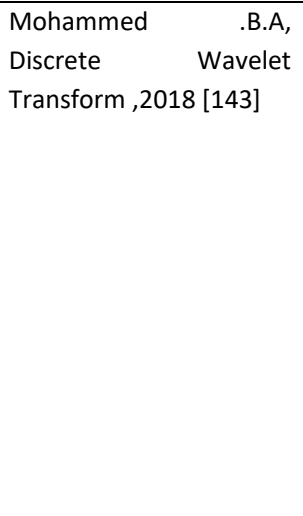 & $\begin{array}{l}\text { MRI/PET. } \\
\text { Diseases: (Coronal, } \\
\text { Sagittal and Trans- } \\
\text { axial) for } \\
\text { Alzheimer's } \\
\text { disease. }\end{array}$ & Averaging method. & $\begin{array}{l}\text { The MRI and PET images } \\
\text { have taken input for the } \\
\text { system. PET image is } \\
\text { decomposed into IHS } \\
\text { transform and the high } \\
\text { activity area is } \\
\text { transforming towards } \\
\text { low activity region. Later } \\
\text { on the transformation } \\
\text { combine the high } \\
\text { frequency coefficient of } \\
\text { MRI and PET image using } \\
\text { the averaging method. }\end{array}$ & $\begin{array}{l}\text { Less edge preserved with } \\
\text { problem of shift invariant } \\
\text { due to wavelet method. }\end{array}$ \\
\hline 11. & $\begin{array}{l}\text { Nsrin Amini, NSCT ,2014 } \\
\text { [144] }\end{array}$ & $\begin{array}{l}\text { MRI/PET. } \\
\text { Diseases: (Coronal, } \\
\text { Sagittal and Trans- } \\
\text { axial) for } \\
\text { Alzheimer's } \\
\text { disease. }\end{array}$ & $\begin{array}{l}\text { maximal energy } \\
\text { rule, maximal } \\
\text { variance rule }\end{array}$ & $\begin{array}{l}\text { The images decomposed } \\
\text { with Nonsubsampled } \\
\text { Contourlet Transform } \\
\text { and then two images } \\
\text { were fused with applying } \\
\text { fusion rules. The } \\
\text { coefficients of the low } \\
\text { frequency band are } \\
\text { combined by a maximal } \\
\text { energy rule and } \\
\text { coefficients of the high } \\
\text { frequency bands are } \\
\text { combined by a maximal } \\
\text { variance rule. }\end{array}$ & $\begin{array}{l}\text { High computational time } \\
\text { with subjective analysis: } \\
\text { blurry of informative } \\
\text { edges. }\end{array}$ \\
\hline
\end{tabular}

\section{Conclusion}

Brain is one of the important organs that have more details like soft and hard tissues with small size that shown in imaging scan therefore the researcher tried to minimize the artifacts for those images with provide high visual quality reading by radiologists. Due to it consider as a main human organ body and effect on patient's health. By the way, the multimodal fusion enhanced the reliability/robust of the information in the medical imaging, improving the accuracy of the diagnosing and obtain a more accurate and clearer description of the anatomy, cells, hard/soft tissues of brain.

This paper presented a critical survey of data fusion state of the art methodologies. Data fusion is a multidisciplinary research field with a potential application of medical images (brain diseases diagnosing).Despite of proposed various medical images fusion techniques in literature with different obstacles in the fused images, at the present time, there are still many open-ended problems in different applications due to image 'artifacts or modalities issues. Extensive use of digital imaging in medicine today, the quality of digital medical images has becomes an important issue. Different types of images 
Ahmed Sabeeh Yousif, Zaid Bin Omar, Usman Ullah Sheikh; Title: A Survey on Multi-Scale Medical images Fusion Techniques: Brain Diseases. Journal of Biomedical Engineering and Medical Imaging, Volume 7, No 1, February (2020), pp 18-38

have different artifact problems, reducing the accuracy of imaging-based brain diseases diagnosis. How to resolve various artifact problems for different types of images is always a pursuit for researchers.

In this paper survey, multi-scale image fusion techniques in terms of medical image (brain) modalities and anatomy/tissues of study have been introduced. Although, there has been significant progress in the medical image fusion research, the medical image fusion algorithms is limited by the practical clinical implications as imposed by the medical or by exists technical issues in image fusion resulting from image noise, resolution difference between images, inter-image variability between the images, lack of sufficient number of images per modality, high cost of imaging, color information, visibility of tissues and increased computational complexity with increasing image space and time resolution.

Besides the fusion based pixel level methods, the objective evaluation of the fusion methods' performances is also a challenging topic. These fusion quality metrics are divided into two major classes, i.e., metrics requiring a reference or not. For the first class, most of methods focus on measuring the difference between the reference and the fused image more accurately. Some state-of-the-art methods are usually developed based on the perception function of human vision. For the second class, a very important topic is how to measure the complementary information (including the complementary, global contrast, etc.) and the visual artifacts appeared in the fused image (including edge, color, visibility of organ in brain diseases).

For our view, the important of brain diseases diagnosing require precisely a robust algorithms according to combining a presented fused image to doctor with minimize artifacts like blur, noise, less visibility, color distortion , preserved edge and complexity ) with a new evaluation metric like to compute and measure the tissues 'information, for example ( WM (white matter) and GM(gray matter) tissues volumes and compute an affected or not by alzheimer's disease to improve our understanding of brain atrophy due to normal aging).

\section{REFERENCES}

[1] B.V. Dasarathy, Editorial: information fusion in the realm of medical applications - a bibliographic glimpse at its growing appeal, Inform. Fus. 13 (1) (2012) 1-9.

[2] Wu, R.-T., \& Jahanshahi, M. R. (2018). Data fusion approaches for structural health monitoring and system identification: Past, present, and future. Structural Health Monitoring.

[3] C. Barillot, D. Lemoine, L.L. Briquer, F. Lachmann, B. Gibaud, Data fusion in medical imaging: merging multimodal and multipatient images, identification of structures and 3D display aspects, Euro. J. Radiol. 17 (1) (1993) 22-27.

[4] S. Marshall, G. Matsopoulos, Morphological data fusion in medical imaging, in: IEEE Winter Workshop on Nonlinear Digital Signal Processing, 1993, IEEE, 1993, pp. 6-1.

[5] G. Matsopoulos, S. Marshall, J. Brunt, Multiresolution morphological fusion of MR and CT images of the human brain, IEE Proc. Vis., Image Signal Process., vol. 141, IET, 1994, pp. 137-142.

[6] J. Julow, T. Major, M. Emri, I. Valalik, S. Sagi, L. Mangel, G. Nemeth, L. Tron, G. Varallyay, D. Solymosi, et al., The application of image fusion in stereotactic brachytherapy of brain tumours, Acta Neurochirurgica 142 (11) (2000) $1253-1258$. 
[7] M.C. Dastjerdi, A. Karimian, H. Afarideh, A. Mohammadzadeh, FMDIB: a software tool for fusion of MRI and DHCSPECT images of brain, in: World Congress on Medical Physics and Biomedical Engineering, September 7-12, 2009, Munich, Germany, Springer, 2009, pp. 741-744.

[8] Slichter, C.P., 2013. Principles of magnetic resonance, 1. Springer Science \& Business Media.

[9] James, A.P., Dasarathy, B.V.: Medical image fusion: a survey of the state of the art. Inf. Fusion 19, 4-19 (2014).

[10] Talbar S.N., Chavan S.S., Pawar A. (2019) Non-subsampled Complex Wavelet Transform Based Medical Image Fusion. In: Arai K., Bhatia R., Kapoor S. (eds) Proceedings of the Future Technologies Conference (FTC) 2018. FTC 2018. Advances in Intelligent Systems and Computing, vol 880. Springer, Cham.

[11] Meher, B., Agrawal, S., Panda, R., \& Abraham, A. (2018). A survey on region based image fusion methods . Information Fusion. doi:10.1016/j.inffus.2018.07.010.

[12] Rajiv Singh, "Medical image fusion: applications, approaches and evaluation", in Proceedings of International Conference on Medical Imaging and Diagnosis, Chicago, USA, October 20-21, 2016.

[13] B.V. Dasarathy, Editorial: information fusion in the realm of medical applications - a bibliographic glimpse at its growing appeal, Inform. Fus. 13 (1) (2012) 1-9.

[14] Pradeep K. Atrey, and M. Anwar Hossain, "Multimodal Fusion for Multimedia Analysis: A Survey", Multimedia Systems, DOI: 10.1007/s00530-010-0182-0, Springer Verlag, 2010.

[15] S.L. Jany Shabu, Dr.C. Jayakumar, T. Surya," Survey of Image Fusion Techniques for Brain Tumor Detection" , International Journal of Engineering and Advanced Technology (IJEAT),2013.

[16] A.P. James, and B.V. Dasarathy, "Medical Image Fusion: A survey of the State of the Art", Information Fusion, vol. 19, pp. 4-19, 2014.

[17] K.P.Indira, and R.Rani Hemamalini, "Analysis on Image Fusion Techniques for Medical Applications", International Journal of Advanced Research in Electrical, Electronics and Instrumentation Engineering, vol. 3, Issue 9, 2014.

[18] Harmandeep Kaur, Er. Jyoti Rani, “Analytical Comparison of Various Image Fusion Techniques” International Journal of Advanced Research in Computer Science and software Engineering, Vol. 5, Issue 5, 2015.

[19] Jasmeetkaur, Er. Rajdavinder, "An Evaluation on Different Image Fusion Techniques", IPASJ International Journal of Computer Science (IIJCS), vol. 2, Issue 4, 2014.

[20] Wu D., Yang A., Zhu L., Zhang C. (2014) Survey of Multi-sensor Image Fusion. In: Ma S., Jia L., Li X., Wang L., Zhou H., Sun X. (eds) Life System Modeling and Simulation. ICSEE 2014, LSMS 2014. Communications in Computer and Information Science, vol 461. Springer, Berlin, Heidelberg.

[21] Fatma E. El-Gamal, and Mohammed Elmogy, "Current Trends in Medical Image Registration and Fusion", Egyptian Informatics Journal, 2015.

[22] S.L. Jany Shabu, Dr.C. Jayakumar, T. Surya," Survey of Image Fusion Techniques for Brain Tumor Detection" , International Journal of Engineering and Advanced Technology (IJEAT),2013.

[23] Bhavana. V, Krishnappa. H.K," A Survey on Multi - Modality Medical Image Fusion", peer-reviewed and accepted to be presented at the IEEE WiSPNET 2016 conference,IEEE,2016. 
Ahmed Sabeeh Yousif, Zaid Bin Omar, Usman Ullah Sheikh; Title: A Survey on Multi-Scale Medical images Fusion Techniques: Brain Diseases. Journal of Biomedical Engineering and Medical Imaging, Volume 7, No 1, February (2020), pp 18-38

[24] Heba M. Elhoseny, El-Sayed Mahmoud El-Rabaie, Osama S. Farag Allah, Fathi E. Abd El-Samie." Medical Image Fusion: A Literature Review Present Solutions and Future Directions", Minufiya J. of Electronic Engineering Research (MJEER), Vol. 26, No. 2, July 2017.

[25] Bikash Meher, Sanjay Agrawal, Rutuparna Panda, Ajith Abraham, A survey on region based image fusion methods , Information Fusion (2018), doi: https://doi.org/10.1016/j.inffus.2018.07.010.

[26] http://www.med.harvard.edu/AANLIB/home.html.

[27] Y. Liu, S. Liu, Z. Wang, A general framework for image fusion based on multi-scale transform and sparse representation, Information Fusion 24 (1) (2015) 147-164.

[28] Z. Xu, Medical image fusion using multi-level local extrema, Inf. Fusion 19 (2014) 38-48.

[29] G.Vivone, L. Alparone, J. Chanussot, M. Dalla Mura, A. Garzelli, G. Licciardi, R. Restaino, Wald, A critical comparison among pansharpening algorithms, IEEE Transactions on Geoscience and Remote Sensing 53 (5) (2015) 2565-2586.

[30] Susmitha Vekkot, and Pancham Shukla, "A Novel Architecture for Wavelet based Image Fusion" Journal of World Academy of Science, Engineering and Technology, Vol. 57, pp. 32-3, 2009.

[31] Peng Geng, Xing Su, Tan Xu and Jianshu Liu, "\Multi-modal Medical Image Fusion Based on the Multiwavelet and Nonsubsampled Direction Filter Bank", International Journal of Signal Processing, Image Processing and Pattern Recognition Vol.8, No.11, pp.75-84 2015.

[32] Maruturi Haribabu , CH.Hima Bindu, Dr.K.Satya Prasad, "A New Approach of Medical Image Fusion using Discrete Wavelet Transform”, ACEEE International Journal on Signal \& Image Processing, Vol. 4, No. 2, May 2013.

[33] Q.P. Zhang, M. Liang, W.C. Sun, 2004, Medical diagnostic image fusion based on feature mapping wavelet neural networks, 2004 IEEE First Symposium on Multi-Agent Security and Survivability, pp. 51-54.

[34] V.D. Calhoun, T. Adali, Feature-based fusion of medical imaging data, IEEE Trans. Inf. Technol. Biomed. 13 (5) (2009) 711-720.

[35] Bengueddoudj, A., Messali, Z., Mosorov, V.: A novel image fusion algorithm based on 2D scale-mixing complex wavelet transform and Bayesian MAP estimation for multimodal medical images.

[36] J. Innov. Opt. Health Sci. 10, 1750001 (2017) , Bengueddoudj, A., Messali, A.: An efficient algorithm for multimodal medical image fusion based on feature selection and PCA using DTCWT (FSPCA-DTCWT). Med. Technol. J. 2 (1), 179-192 (2018).

[37] Ganasala, P., Kumar, V.: CT and MR image fusion scheme in nonsubsampled contourlet transform domain. J. Digit. Imag. 27, 407-418 (2014).

[38] Q. Guihong, Z. Dali, and Y. Pingfan. Medical image fusion by wavelet transform modulus maxima. Optics Express, 9(4):184-190, 2001.

[39] Y. Kirankumar and S. Devi. Transform-based medical image fusion. International Journal of Biomedical Engineering and Technology, 1(1):101-110, 2007. 
[40] L. Xu, S. Zheng, J. Jia, Unnatural LO sparse representation for natural image deblurring, in: 2013 IEEE Computer Society Conference on Computer Vision and Pattern Recognition(CVPR 2013), Portland, OR, USA, June 23-28, 2013, 2013, pp. 1107-1114.

[41] Udhaya Suriya TS, Rangarajan P "Brain tumour detection using discrete wavelet transform based medical image fusion" Biomedical Research, 28 (2), 2017, pp. 684-688.

[42] J. Yang, J. Wright, T. S. Huang, Y. Ma, Image super-resolution as sparse representation of raw image patches, in: 2008 IEEE Computer Society Conference on Computer Vision and Pattern Recognition (CVPR 2008), 24-26 June 2008, Anchorage, Alaska, USA, 2008.

[43] B. Yang, S. Li, Multifocus image fusion and restoration with sparse representation, IEEE T. Instrumentation and Measurement 59 (4) (2010) 884-892.

[44] S. Akbar, R. Naqvi, I. Touqir, A. M. Siddiqui, Sparse representation of image and video using easy path wavelet transform, Signal Processing 103 (2014) 331-347.

[45] S.A. Kuhn, B. Romeike, J. Walter, R. Kal, R. Reichart, Multiplanar MRI-CT fusion neuronavigation-guided serial stereotactic biopsy of human brain tumors: proof of a strong correlation between tumor imaging and histopathology by a new technical approach, J. Can. Res. Clin. Oncol. 135 (9) (2009) 1293-1302.

[46] V. Barra, J.-Y. Boire, Automatic segmentation of subcortical brain structures in MR images using information fusion, IEEE Trans. Med. Imag. 20 (7) (2001) 549-558.

[47] D. Dey, D.G. Gobbi, P.J. Slomka, K.J. Surry, T.M. Peters, Automatic fusion of freehand endoscopic brain images to three-dimensional surfaces: creating stereoscopic panoramas, IEEE Trans. Med. Imag. 21 (1) (2002) 23-30.

[48] H. Lee, H. Hong, Hybrid surface-and voxel-based registration for MR-PET brain fusion, in: Image Analysis and Processing-ICIAP 2005, Springer, 2005, pp. 930-937.

[49] Y. Xia, S. Eberl, D. Feng, Dual-modality 3D brain PET-CT image segmentation based on probabilistic brain atlas and classification fusion, in: 17th IEEE International Conference on Image Processing (ICIP), 2010, IEEE, 2010, pp. 25572560 .

[50] J.-D. Lee, B.-R. Huang, C.-H. Huang, A surface-projection MMI for the fusion of brain MR and SPECT images, in: IEEE Region 10 Conference, 2004, TENCON 2004, IEEE, 2004, pp. 179-182.

[51] M.C. Dastjerdi, A. Karimian, H. Afarideh, A. Mohammadzadeh, FMDIB: a software tool for fusion of MRI and DHCSPECT images of brain, in: World Congress on Medical Physics and Biomedical Engineering, September 7-12, 2009, Munich, Germany, Springer, 2009, pp. 741-744.

[52] Rajesh Kumar P., Arun Prasath T., Pallikonda Rajasekaran M., Vishnuvarthanan G. (2019) Brain Subject Segmentation in MR Image for Classifying Alzheimer's Disease Using AdaBoost with Information Fuzzy Network Classifier. In: Nayak J., Abraham A., Krishna B., Chandra Sekhar G., Das A. (eds) Soft Computing in Data Analytics. Advances in Intelligent Systems and Computing, vol 758. Springer, Singapore.

[53] Blank R.H. (2019) Alzheimer's Disease and Other Dementias: An Introduction. In: Social \& Public Policy of Alzheimer's Disease in the United States. Palgrave Pivot, Singapore.

[54] Khanal B, Lorenzi M, Ayache N, Pennec X (2016) A biophysical model of brain deformation to simulate and analyze longitudinal MRIs of patients with Alzheimer's disease. Neurolmage 134:35-52. 
Ahmed Sabeeh Yousif, Zaid Bin Omar, Usman Ullah Sheikh; Title: A Survey on Multi-Scale Medical images Fusion Techniques: Brain Diseases. Journal of Biomedical Engineering and Medical Imaging, Volume 7, No 1, February (2020), pp 18-38

[55] Tokuchi R, Hishikawa N, Sato K, Hatanaka N, Fukui Y, TakemotoaM, Ohta Y, Yamashita T, Abe K (2016) Agedependent cognitive and affective differences in Alzheimer's and Parkinson's diseases in relation to MRI findings. J Neurol Sci 365:3-8.

[56] Rajesh Kumar P., Arun Prasath T., Pallikonda Rajasekaran M., Vishnuvarthanan G. (2019) Decisive Tissue Segmentation in MR Images: Classification Analysis of Alzheimer's Disease Using Patch Differential Clustering. In: Kulkarni A., Satapathy S., Kang T., Kashan A. (eds) Proceedings of the 2nd International Conference on Data Engineering and Communication Technology. Advances in Intelligent Systems and Computing, vol 828. Springer, Singapore.

[57] Mirzaei G, Adeli A, Adeli H (2016) Imaging and machine learning techniques for diagnosis of Alzheimer's disease. Rev Neurosci 27(8):857-870. https://doi.org/10.1515/revneuro-2016-0029.

[58] A.P. James, and B.V. Dasarathy, "Medical Image Fusion: A survey of the State of the Art", Information Fusion, vol. 19, pp. 4-19, 2014.

[59] OBREJA, Cristian, Dragoș.(2018). Useful information analysis from medical images. Diagnosis improvement by hemodynamic modeling and processing (Doctoral dissertation).Retrievedfrom https://www.arthra.ugal.ro/bitstream/handle/123456789/5044/Rezumat Eng Tez a_doctorat_Cristian_Obreja_2018.pdf?sequence=2\&isAllowed=y.

[60] C. He, Q. Liu, H. Li, et al., Multimodal medical image fusion based on IHS and PCA, Proc. Eng. 7 (2010) 280-285.

[61] S. Daneshvar, H. Ghassemian, MRI and PET image fusion by combining IHS and retina-inspired models, Inf. Fusion 11 (2) (2010) 114-123.

[62] Matsopoulos, G.K., Marshall, S., Brunt, J.N.H. 1994. Multiresolution morphological fusion of MR and CT images of the human brain, IEE Proceedings of Visual Image Signal Processing, vol. 141 (3), pp. 137-142.

[63] Patil, U., Mudengudi, U. 2011. Image fusion using hierarchical PCA, Proceedings of Internal Conference Image Information Processing, pp. 1-6.

[64] Q. Guihong, Z. Dali, Y. Pingfan, Medical image fusion by wavelet transform modulus maxima, Opt. Express 9 (4) (2001) 184-190.

[65] R. Singh, A. Khare, Fusion of multimodal medical images using Daubechies complex wavelet transform-A multiresolution approach, Inf. Fusion (2012).

[66] Y. Zheng, A.E. Essock, B.C. Hansen, et al., A new metric based on extended spatial frequency and its application to DWT based fusion algorithms, Inf. Fusion 8 (2) (2007) 177-192.

[67] Singh, R., Vatsa, M., Noore, A. 2009. Multimodal medical image fusion using redundant discrete wavelet transform, In: IEEE Seventh International Conference on Advances in Pattern Recognition, 2009, pp. 232-235.

[68] Liu, Y., Yang, J., Sun, J. 2010. PET/CT medical image fusion algorithm based on multiwavelet transform, In: IEEE 2nd International Conference on Advanced Computer Control, 2010, pp. 264-268.

[69] Xue-jun, W., Ying, M. 2010. A medical image fusion algorithm based on lifting wavelet transform, In: IEEE International Conference on Artificial Intelligence and Computational Intelligence, 2010 (3), pp. 474-476. 
[70] L. Yang, B.L. Guo, W. Ni, Multimodality medical image fusion based on multiscale geometric analysis of contourlet transform, Neurocomputing 72 (1) (2008) 203-211.

[71] G. Bhatnagar, Q.M. Wu, Z. Liu, Directive contrast based multimodal medical image fusion in NSCT domain, IEEE Trans. Multimed. 15 (5) (2013) 1014-1024.

[72] Q. Miao, C. Shi, P. Xu, et al., A novel algorithm of image fusion using shearlets, Opt. Commun. 284 (6) (2011) 15401547.

[73] L. Wang, B. Li, L. Tian, Multi-modal medical volumetric data fusion using 3D discrete shearlet transform and globalto-local rule, IEEE Trans. Biomed. Eng. (2014).

[74] H. Yin, S. Li, L. Fang, Simultaneous image fusion and super-resolution using sparse representation, Inf. Fusion 14 (3) (2013) 229-240.

[75] B. Yang, S. Li, Pixel-level image fusion with simultaneous orthogonal matching pursuit, Inf. Fusion 13 (1) (2012) 10-19.

[76] H. Yin, S. Li, Multimodal image fusion with joint sparsity model, Opt. Eng. 50 (6) (2011) 7-10.

[77] N. Yu, T. Qiu, F. Bi, et al., Image features extraction and fusion based on joint sparse representation, IEEE J. Sel. Top. Signal Process. 5 (5) (2011) 1074-1082.

[78] S. Li, H. Yin, L. Fang, Group-sparse representation with dictionary learning for medical image denoising and fusion, IEEE Trans. Biomed. Eng. 59 (12) (2012) 3450-3459.

[79] R.Redondo,F.Sroubek,S.Fischer, and G.Grstobal," Multifoucs image fusion using the log-Gabor transform and a multisize windows technique"Inf .Fusion, Vol .10.no.2,pp.163-171,2009.

[80] S. Li, X. Kang X, J. Hu, Image fusion with guided filtering, IEEE Trans. Image Process. 22 (7) (2013) $2864-2875$.

[81] Z. Xu, Medical image fusion using multi-level local extrema, Inf. Fusion 19 (2014) 38-48.

[82] R. Shen, I. Cheng, A. Basu, Cross-scale coefficient selection for volumetric medical image fusion, IEEE Trans. Biomedical Engineering 60 (4) (2013) 1069-1079

[83] Y. Liu, S. Liu, Z. Wang, A general framework for image fusion based on multi-scale transform and sparse representation, Information Fusion 24 (1) (2015) 147-164.

[84] [ Aggarwal, Jakek,ed." Multi sensor fusion for computer vision”. Vol.99. Springer sciences \& Business media, 2013.

[85] , C. Pohl, J Van Genderen, “ Multisensor Image Fusion of remotely sensed data : concepts, methods and applications", International Journal Remote Sensing vol19(5) march 2009.

[86] S. S. Hana, b, * , H.T. Lia, H.Y.Gua,b,"'” The study on image fusion for high spatial resolution remote sensing images", The international Archives of the photogrammetry, Remote sensing and spatial information sciences vol XXXVII. Part B7. Beijing, April 2008.

[87] Jagalingam P, ArkalVittal Hedge. "Pixel Level Image Fusion- A Review on various Techniques.

[88] Z. Xu, Medical image fusion using multi-level local extrema, Inf. Fusion 19 (2014) 38-48. 
Ahmed Sabeeh Yousif, Zaid Bin Omar, Usman Ullah Sheikh; Title: A Survey on Multi-Scale Medical images Fusion Techniques: Brain Diseases. Journal of Biomedical Engineering and Medical Imaging, Volume 7, No 1, February (2020), pp 18-38

[89] A. A. Goshtasby, S. Nikolov, Image fusion: Advances in the state of the art, Information Fusion 8 (2) (2007) 114118.

[90] R.Redondo,F.Sroubek,S.Fischer, and G.Grstobal," Multifoucs image fusion using the log-Gabor transform and a multisize windows technique"Inf .Fusion, Vol .10.no.2,pp.163-171,2009.

[91] Z. Zhang, R. S. Blum, A categorization of multiscale-decomposition-based image fusion schemes 645 with a performance study for a digital camera application, Proceedings of the IEEE 87 (8) (1999) 1315-1326.

[92] G. Pajares, J. M. de la Cruz, A wavelet-based image fusion tutorial, Pattern Recognition 37 (9) 660 (2004) 18551872

[93] J. J. Lewis, R. J. O. Callaghan, S. G. Nikolov, D. R. Bull, N. Canagarajah, Pixel- and regionbased image fusion with complex wavelets, Information Fusion 8 (2) (2007) 119-130.

[94] E. J. Cands, D. L. Donoho, Curvelets and curvilinear integrals, Journal of Approximation Theory 113 (1) (2001) 5990.

[95] F. Nencini, A. Garzelli, S. Baronti, L. Alparone, Remote sensing image fusion using the curvelet transform, Information Fusion 8 (2) (2007) 143-156, special Issue on Image Fusion: Advances in the State of the Art..

[96] B. Yang, S. Li, Multifocus image fusion and restoration with sparse representation, IEEE Trans730 actions on Instrumentation and Measurement 59 (4) (2010) 884-892.

[97] Y. C. Pati, R. Rezaiifar, P. S. Krishnaprasad, Orthogonal matching pursuit: recursive function approximation with applications to wavelet decomposition, in: Proceedings of Asilomar Conference on Signals, Systems and Computers, Vol. 1, 1993, pp. 40-44.

[98] S. Li, H. Yin, L. Fang, Group-sparse representation with dictionary learning for medical image 735 denoising and fusion, IEEE Transactions on Biomedical Engineering 59 (12) (2012) 3450-3459.

[99] C. Chen, Y. Li, W. Liu, J. Huang, Image fusion with local spectral consistency and dynamic gradient sparsity, in: Proceedings of IEEE Conference on Computer Vision and Pattern Recognition, 2014, pp. 2760-2765.

[100] B. Yang, S. Li, Pixel-level image fusion with simultaneous orthogonal matching pursuit, Infor740 mation Fusion 13 (1) (2012) 10-19.

[101] H. Yin, S. Li, Multimodal image fusion with joint sparsity model, Optical Engineering 50 (6) (2011) $067007.1-$ 067007.10.

[102] N. Yu, T. Qiu, F. Bi, A. Wang, Image features extraction and fusion based on joint sparse representation, IEEE Journal of Selected Topics in Signal Processing 5 (5) (2011) 1074-1082.

[103] B.yang.J. Luo, S. Li, Color image fusion with extend joint sparse model, in: Proceedings of International Conference on Pattern Recognition, 2012, pp. 376-379.

[104] J. Luo, S. Li, Color image fusion with extend joint sparse model, in: Proceedings of International Conference on Pattern Recognition, 2012, pp. 376-379.

[105] H. Yin, S. Li, L. Fang, Simultaneous image fusion and super-resolution using sparse representa750 tion, Information Fusion 14 (3) (2013) 229-240 . 
[106] S. Li, H. Yin, L. Fang, Remote sensing image fusion via sparse representations over learned dictionaries, IEEE Transactions on Geoscience and Remote Sensing 51 (9) (2013) 4779-4789.

[107] M. Kim, D. K. Han, H. Ko, Joint patch clustering-based dictionary learning for multimodal image fusion, Information Fusion 27 (1) (2016) 198-214.

[108] W. Wang, L. Jiao, S. Yang, Fusion of multispectral and panchromatic images via sparse representation and local autoregressive model, Information Fusion 20(1) (2014) 73-87.

[109] H. R. Shahdoosti, H. Ghassemian, Combining the spectral PCA and spatial PCA fusion methods by an optimal filter, Information Fusion 27 (1) (2016) 150-160.

[110] T. M. Tu, S. C. Su, H. C. Shyu, P. S. Huang, A new look at IHS-like image fusion methods, Information Fusion 2 (3) (2001) 177-186.

[111] T. M. Tu, S. C. Su, H. C. Shyu, P. S. Huang, A new look at IHS-like image fusion methods, Information Fusion 2 (3) (2001) 177-186.

[112] T. M. Tu, P. S. Huang, C. L. Hung, C. P. Chang, A fast intensity-hue-saturation fusion technique 800 with spectral adjustment for IKONOS imagery, IEEE Geoscience and Remote Sensing Letters 1 (4) (2004) 309-312.

[113] S. Rahmani, M. Strait, D. Merkurjev, M. Moeller, T. Wittman, An adaptive IHS pan-sharpening method, IEEE Geoscience and Remote Sensing Letters 7 (4) (2010) 746-750.

[114] Anjali Malviya, S. G. Bhirud, "Image Fusion of Digital Images", International Journal of Recent Trends in Engineering, Vol. 2, No. 3, November 2009.

[115] J. J. Lewis, J. Robert, O. Callaghan, S. G. Nikolov, D. R. Bull and N. Canagaraja, "Pixel- and region-based image fusion with complex wavelets," Information Fusion, Elsevier, vol. 8, pp. 119-130, 2007.

[116] N. Mitianoudis and T. Stathaki, "Pixel-based and Region-based Image Fusion schemes using ICA bases," Elsevier Information Fusion, vol. 8, pp. 131-142, 2007.

[117] N. Cvejic, Bull, DR and N. Canagarajah, "Regionbased multimodal image fusion using ICA bases," IEEE Sensors Journal, vol. 7, no. 5, pp. 743- 751, 2007.

[118] Susmitha Vekkot, and Pancham Shukla, "A Novel Architecture for Wavelet based Image Fusion" Journal of World Academy of Science, Engineering and Technology, Vol. 57, pp. 32-3, 2009

[119] School of Computer Science, Fudan University Shanghai, china "Information Fusion" www.elsevier.com/locate/inffus.

[120] Mamta Sharma, Geetanjali institute of technical studies, Udaipur, India "A Review : Image Fusion Techniques and Applications Vol. 7(3), 2016 1082- 1085

[121] Zheng, M. M., Krishnan, S. M., \& Doraiswami, R. (n.d.). Data fusion application for computer-assisted clinical endoscopic image analysis. IEEE CCECE2002. Canadian Conference on Electrical and Computer Engineering. Conference Proceedings (Cat. No.02CH37373). doi:10.1109/ccece.2002.1013103.

[122] G. Piella, A general framework for multiresolution image fusion: from pixels to regions, Information Fusion 4 (4) (2003) 259-280. 
Ahmed Sabeeh Yousif, Zaid Bin Omar, Usman Ullah Sheikh; Title: A Survey on Multi-Scale Medical images Fusion Techniques: Brain Diseases. Journal of Biomedical Engineering and Medical Imaging, Volume 7, No 1, February (2020), pp 18-38

[123] [ S. Li, X. Kang, J. Hu, Image fusion with guided filtering, IEEE Trans. Image Process. 22 (7) (2013) $2864-2875$.

[124] A. Toet, Image fusion by a ratio of low-pass pyramid, Pattern Recognit. Lett. 9 (4) (1989) 245-253.

[125] P.J. Burt, E.H. Adelson, The Laplacian pyramid as a compact image code, IEEE Trans. Commun. 31 (4) (1983) $532-$ 540.

[126] Z. Wang, A.C. Bovik, H.R. Sheikh, et al., Image quality assessment: from error visibility to structural similarity, IEEE Trans. Image Process. 13 (4) (2004) 600-612.

[127] Q. Miao, C. Shi, P. Xu, et al., A novel algorithm of image fusion using shearlets, Opt. Commun. 284 (6) (2011) 15401547, M. Hossny, S. Nahavandi, D. Creighton, Comments on 'Information measure for performance of image fusion', Electron. Lett. 44 (18) (2008) 1066-1067.

[128] A. Mittal, R. Soundararajan, A.C. Bovik, Making a "completely blind" image quality analyzer, IEEE Trans. Signal Process. Lett. 20 (3) (2013) 209-212., J.H. Jang, Y. Bae, J.B. Ra, Contrast-enhanced fusion of multisensor images using subband-decomposed multiscale retinex, IEEE Trans. Image Process. 21 (8) (2012) 3479-3490.

[129] J.H. Jang, Y. Bae, J.B. Ra, Contrast-enhanced fusion of multisensor images using subband-decomposed multiscale retinex, IEEE Trans. Image Process. 21 (8) (2012) 3479-3490.

[130] A. Mittal, R. Soundararajan, A.C. Bovik, Making a "completely blind" image quality analyzer, IEEE Trans. Signal Process. Lett. 20 (3) (2013) 209-212.

[131] Z. Xu, Medical image fusion using multi-level local extrema, Inf. Fusion 19 (2014) 38-48.

[132] Q. Miao, C. Shi, P. Xu, et al., A novel algorithm of image fusion using shearlets, Opt. Commun. 284 (6) (2011) 15401547.

[133] H.R. Sheikh, A.C. Bovik, Image information and visual quality, IEEE Trans. Image Process. 15 (2) (2006) $430-444$.

[134] J.Choi,K.Yu, Y. Kim, A new adaptive component-substitution-based satellite image fusion by 805 using partial replacement, IEEE Transactions on Geoscience and Remote Sensing 49 (1) (2011) 295-309.

[135] Bhatnagar, G., Wu, Q. M. J., \& Liu, Z. (2013). Directive Contrast Based Multimodal Medical Image Fusion in NSCT Domain. IEEE Transactions on Multimedia, 15(5), 1014-1024. doi:10.1109/tmm.2013.2244870.

[136] Yang, G., Li, M., Chen, L., \& Yu, J. (2015). The Nonsubsampled Contourlet Transform Based Statistical Medical Image Fusion Using Generalized Gaussian Density. Computational and Mathematical Methods in Medicine, 2015, 1-13. doi:10.1155/2015/262819.

[137] Du, J., Li, W., Xiao, B., \& Nawaz, Q. (2016). Union Laplacian pyramid with multiple features for medical image fusion. Neurocomputing, 194, 326-339. doi:10.1016/j.neucom.2016.02.047.

[138] Zhu, Z., Chai, Y., Yin, H., Li, Y., \& Liu, Z. (2016). A novel dictionary learning approach for multi-modality medical image fusion. Neurocomputing, 214, 471-482. doi:10.1016/j.neucom.2016.06.036.

[139] Chen, C.-I. (2017). Fusion of PET and MR Brain Images Based on IHS and Log-Gabor Transforms. IEEE Sensors Journal, 17(21), 6995-7010. doi:10.1109/jsen.2017.2747220. 
[140] Du, J., Li, W., \& Xiao, B. (2017). Anatomical-Functional Image Fusion by Information of Interest in Local Laplacian Filtering Domain. IEEE Transactions on Image Processing, 26(12), 5855-5866. doi:10.1109/tip.2017.2745202.

[141] Daniel, E. (2018). Optimum Wavelet-Based Homomorphic Medical Image Fusion Using Hybrid Genetic-Grey Wolf Optimization Algorithm. IEEE Sensors Journal, 18(16), 6804-6811. doi:10.1109/jsen.2018.2822712.

[142] Rajalingam B, Priya R,2018, Multimodal Medical Image Fusion Using Various Hybrid Fusion Techniques For clinical Treatment Analysis, Open Journal Systems, Smart Construction Research, Volume 2 Issue 4 | 2018.

[143] Du, J., Li, W., \& Xiao, B. (2018). Fusion of anatomical and functional images using parallel saliency features. Information Sciences, 430-431, 567-576. doi:10.1016/j.ins.2017.12.008.

[144] Abdulkareem, M. B. (2018). Design and Development of Multimodal Medical Image Fusion using Discrete Wavelet Transform. 2018 Second International Conference on Inventive Communication and Computational Technologies (ICICCT). doi:10.1109/icicct.2018.8472997.

[145] M.M. Fraz, P. Remagnino, A. Hoppe, S. Barman, Retinal image analysis aimed at extraction of vascular structure using linear discriminant classifier, in: International Conference on Computer Medical Applications (ICCMA), 2013, DOI:10.1109/ICCMA.2013.6506180 